\title{
Long-term results of heart transplant in recipients older and younger than 65 years: a comparative study of mortality, rejections, and neoplasia in a cohort of 445 patients
}

\author{
M.G. Crespo-Leiro, M.J. Paniagua-Martín, J. Muñiz, R. Marzoa, P. Piñón, J.A. \\ Rodríguez, L.F. Hermida, R. Calviño, J.J. Cuenca, b, A. Juffé, A. Castro-Beiras
}

\begin{abstract}
Background. Whether being older than 65 years should be considered an absolute counterindication to heart transplant (HT), as it is in some centers, is controversial. In our centre, patients older than 65 years are accepted for HT if they satisfy stringent conditions. The aim of this study was to examine whether heart recipients older than 65 years have a greater risk of rejection, neoplasia, or mortality than younger ones.

Methods. We studied 445 patients who underwent HT between April 1991 and December 2003, 42 of whom were older than 65 years and 403 who were 65 years or younger. The parameters evaluated were the cumulative incidences of neoplasias and rejections (ISHLT grade $\geq 3 \mathrm{~A}$ ), and the survival rates 1 month, 1 year, and 5 years post-HT.

Results. The two groups had similar percentages of patients with at least one rejection episode ( $\leq 65$ years $56.9 \%,>65$ years $51.3 \% ; P>.05)$, and although there were proportionally almost twice as many tumors in the older group $(14.2 \%)$ as in the younger $(7.9 \%)$, this difference was not statistically significant either. Nor were there any significant differences in survival, the 1-month, 1-year, and 5-year rates being $87.8 \%, 82.1 \%$, and $68.8 \%$, respectively, in the younger group and $85.7 \%, 78.6 \%$, and $73.4 \%$, respectively, in the older.

Conclusions. Among carefully selected patients aged more than 65 years, HT can be performed without incurring greater risk of rejection, malignancy, or death than is found among recipients younger than 65 years.
\end{abstract}

Heart transplantation (HT) has become the therapy of choice for end-stage heart disease, but most HT programs exclude patients older than 65 years because advanced age is a risk factor for morbidity and mortality among heart recipients. ${ }^{1}$ It is possible, however, that properly selected elderly patients may be exceptions to this general rule; although some centers have reported worse prognosis for recipients older than $65,{ }^{2}$ and 3 others have found survival rates to be excellent among over-65s subjected to rigorous selection criteria, ${ }^{4,5}$ and 6 and there have also been reports of recipients older than 70 years. ${ }^{7 \text { and } 8}$

In our center, patients are not excluded from our HT program solely because they are older than $65 .^{9}$ To evaluate our policy, in this study we compared the long-term outcomes of heart recipients younger and older than 65 as regards rejection, neoplasias, and mortality.

\section{Patients and methods}

Between April 1991 and December 2003, 445 orthotopic HTs were performed at the Juan Canalejo Hospital, La Coruña, Spain. In 42 cases $(9.4 \%)$, the patient was older than 65 years. We compared the $\leq 65$ years and $>65$ years groups regarding basal characteristics (age and gender of recipient, transplant urgency [\% of status I patients] donor age, allograft ischemic time, cardiopulmonary bypass time); the cumulative incidence of rejections (defined as shown by an ISHLT biopsy grade of 3A or worse); the cumulative incidence of neoplasias; and death rates 1 month, 1 year, 5 years, and 10 years after transplant.

\section{Statistical Analysis}

Results for categorical variables are summarized as percentages and results for continuous variables as means \pm SD. The significance of differences between group means was estimated by unpaired Student $t$ tests for continous variables and by $\chi^{2}$ tests for discrete variables. The significance of differences between Kaplan-Meier survival curves was estimated by the Wilcoxon rank-sum test. In all comparisons, 
statistical nonsignificance (NS) was deemed to be shown by a $P$ value $>.05$, although a $P$ value between .05 and .10 was considered to indicate a tendency toward significance.

\section{Results}

The $>65$ group was aged $67.52 \pm 1.07$ years (range, 66 to 70 years). There were no significant differences between the older and younger groups regarding height, weight, donor age, ischemic time, or cardiopulmonary bypass time. The number of patients with urgent status at the time of HT was larger in the younger group, $62(15.4 \%)$ versus $1(2.4 \%)$ in the older group $(P<.02)$. The two groups did not differ significantly in incidence of acute rejection: $56.9 \%$ of the younger group and $51.3 \%$ of the older group having suffered at least one episode (NS). The difference in cumulative incidence of neoplasia was also nonsignificant $(7.9 \%$ and $14.2 \%$ in younger and older groups, respectively), and neither were there any differences in survival, the 1-month, 1-year, and 5-year rates being respectively $87.8 \%, 82.1 \%$, and $68.8 \%$ in the younger group and $85.7 \%, 78.6 \%$ and $73.4 \%$ in the older.

\section{Discussion}

The upper age limit for heart transplant is the subject of considerable debate, ${ }^{10}$ a controversy being fueled by the scarcity of donors as the major limiting factor for HT. According to the records of multicenter registries, the risk of death post-HT increases steadily with age at HT among recipients older than 55 years, ${ }^{1}$ but several single-center reports suggest that age has no adverse effect on survival if the patient satisfies rigorous selection criteria. $5,6,8,11$ and 12

In this study, we found that among the 42 patients older than 65 years who received hearts in our center in a period of almost 13 years (April 1991 to December 2003), cumulative morbidity (acute rejections and malignancy) and short- and long-term mortality did not differ significantly from the results for the other 403 HT patients who received hearts in our institution during this period. In selecting patients older than 65 years for HT, we try to avoid the cumulative effect of multiple comorbid conditions. This is reflected by the difference between the older and younger groups as regards the percentage of patients with urgent status, namely, $15.4 \%$ in the latter and only $2.4 \%$ in the former. In studies that have found worse results with older patients, the percentage of patients with ischemic etiology or comorbid conditions such as diabetes mellitus has also been higher in the older group, ${ }^{2}$ and it seems likely that this must have affected the outcome of HT.

In an earlier study of this issue carried out in our center in $1999,{ }^{9}$ we found the 26-member group of over-65s to have suffered fewer rejection episodes than under-65s. In the present study, in which the number of over-65s has grown to 42, no such advantage was observed. Although larger than 6 years ago, the over-65 group is still small, and this is the main limitation of this study. If it had been larger, it seems possible that the difference between the two groups as regards the incidence of neoplasias (in the older group almost double the incidence observed in the younger group) might have proved statistically significant.

\section{Conclusions}

Short- and long-term mortality, and the incidence of rejection and neoplasia, were in this 13-year study no worse among carefully selected heart transplant patients aged over 65 years at transplant than among younger recipients. Chronological age should not be an overriding criterion for the exclusion of patients from HT programs.

\section{References}

1. D. Taylor, L. Edwards, M. Boucek, et al. The Registry of the International Society for Heart and Lung Transplantation: Twenty-first Official Adult Heart Transplant Report-2004. J Heart Lung Transplant, 23 (2004), p. 796.

2. J. Peraira, J. Segovia, R. Fuentes, et al. Differential characteristics of heart transplantation in patients older than 60 years. Transplant Proc, 35 (2003), p. 1959.

3. R. Favaloro, M. Diez, C. Bertolotti, et al. Orthotopic heart transplantation in elderly patients: a 10-year experience at a single center. Transplant Proc, 36 (2004), p. 1692

4. D. Baran, I. Galin, M. Courtney, et al. Cardiac transplantation in the older recipient: excellent long-term survival based on pretransplant screening. Transplant Proc, 35 (2003), p. 2465. 
5. J. Morgan, R. John, A. Weinberg, et al. Long-term results of cardiac transplantation in patients 65 years of age and older: a comparative analysis. Ann Thorac Surg, 76 (2003), p. 1982.

6. P. Demers, S. Moffatt, P. Oyer, et al. Long-term results of heart transplantation in patients older than 60 years. Thorac Cardiovasc Surg, 126 (2003), p. 224.

7. J. Morgan, R. John, A. Weinberg, et al. Heart transplantation in diabetic recipients: a decade review of 161 patients at Columbia Presbyterian. J Thorac Cardiovasc Surg, 127 (2004), p. 1486.

8. C. Blanche, D. Blanche, B. Kearney, et al. Heart transplantation in patients seventy years of age and older: a comparative analysis of outcome. J Thorac Cardiovasc Surg, 121 (2001), p. 532.

9. M. Crespo-Leiro, M. Paniagua, J. Rodriguez, et al. Morbidity and mortality among heart transplant patients older and younger than 65 years. Transplant Proc, 31 (1999), p. 2537.

10. T. Cimato, M. Jessup. Recipient selection in cardiac transplantation: contraindications and risk factors for mortality. J Heart Lung Transplant, 21 (2002), p. 1161.

11. J. Nagendran, S. Wildhirt, D. Modry, et al. A comparative analysis of outcome after heart transplantation in patients aged 60 years and older: the University of Alberta experience. J Card Surg, 19 (2004), p. 559.

12. J. McCarthy, P. McCarthy, M. Massad, et al. Risk factor for death after heart transplantation: does a single-center experience correlate with multicenter registries?. Ann Thorac Surg, 65 (1998), p. 1574. 